\title{
The Most Important Origin of Twist and Tilt of Magnetic Fields in Solar Active Regions
}

\author{
L. Tian \\ Beijing Astronomical Observatory, National Astronomical \\ Observatories, Chinese Academy of Sciences, A20 Datun Road, \\ Chaoyang District, Beijing 100012, China
}

\section{H. Zhang and S. Bao}

\section{Beijing Astronomical Observatory, National Astronomical Observatories, Chinese Academy of Sciences, Beijing, China}

\begin{abstract}
In this paper, we show observed properties of tilt and twist of bipolar active regions magnetic fields, using magnetograph data at Huairou, Beijing Astronomical Observatory. We deduce that origin of the twist of magnetic fields is same as that of tilt of bipolar magnetic fields. In another word, they are primarily produced by Coriolis force acting on a rising flux tube through convection zone.
\end{abstract}

\section{Introduction}

In photospheric magnetograms, bipolar magnetic fields are tilted to solar equator, with the proceeding one close to the equator. On the other hand, the magnetic fields are left/right-handed twist in the northern/southern hemisphere, denoting the nonpotential property of the magnetic fields. The averaged tilt angles of active regions vary with solar latitude. This was shown many years ago (Hale et al. 1919) and confirmed with more recent data (Wang \& Sheeley 1989, Howard 1991), which tells us something about the effects of the Coriolis force on the rising flux tubes. The tilt of bipolar magnetic fields has been explained as the result of the Coriolis force writhing a rising and expending toroidal flux loops. (Babcock 1961; Wang \& Seeley 1991). However, the effect of turbulent convective motions would act to randomize the tilt angles. This can explain fluctuation of the tilt angle to the mean tilt angle (Longcope et al. 1996; Fisher et al. 1995). The observed properties of the tilt angles are thus consistent with the presence of a systematic component due to the Coriolis forces on the expending flux loops, together with a random component associated with turbulent convective motions. On the basis of the definition of the force-free parameter $\alpha_{f}=\frac{(\nabla \times \mathbf{B})_{\|}}{\mathbf{B}_{\|}}=\frac{\mathbf{B}_{\|} \cdot(\nabla \times \mathbf{B})_{\|}}{\mathbf{B}_{\|}{ }^{2}}$ and the longitudinal component of current helicity $<h_{\|}>=<\mathbf{B}_{\|} \cdot(\nabla \times \mathbf{B})_{\|}>$, both of them should mirror the twist of magnetic lines in a subphotospheric flux tube in the view of observations. And, a positive/negative value is corresponding to the right/left-handed twist of magnetic lines. What is the most important origin of the twist of magnetic fields? This subject is the very important to some dynamo and flux tube models. 


\section{Data and Observation}

Data of vector magnetic fields are obtained at Huairou, Beijing Astronomical Observatory, with a field of view about $5.23^{\prime} \times 3.63^{\prime}$. Each magnetogram is the sum of 256 individual frames, corresponding to a temporal resolution of about $5 \mathrm{~min}$ and a spatial resolution of 2 arcsec. The 180-degree azimuthal ambiguity may be resolved according to the potential field approximation method with taking into account the evolution of active regions and the orientation of chromospheric fibrils. After $3 \times 4$ pixels smooth average was made, the noise level is less than 20G for the line-of-sight field and 100G for the transverse field. Magneto-optical effect is insignificant (Wang et al. 1996) in FeI 5324.191 . Several tests were made particularly to compare the measured field azimuth for a few sunspots. The observed azimuth difference is less than $10^{\circ}$. Bao et al. (2000) compared vector magnetogram of an active region between Huairou and Mees Solar Observation and found a qualitative agreement between them. They estimated that Faraday rotation in Huairou magnetogram contributes about $12^{\circ}$.

153 bipolar active regions were used. In these active regions, magnetic fields are simple configuration and adhering to Hale-Nicholson Law. They are isolated from others and located near the central meridian. All vector magnetic field data were acquired with favorable weather and seeing conditions during observation.

\section{Observed Properties of the Tilt Angle and the Twist Parameter}

Tian et al. (1999) found that the averaged tilt angle depends on magnetic flux and separation of magnetic polarity, and on the latitude, called as Joy's Law (Hale et al. 1919). All these results suggest that the dominant tilting occurs from Coriolis Force. The twist of active regions magnetic fields has been obtained by many methods (Martin et al. 1994; Rust \& Kumar 1996; Pevtsove et al. 1995; Bao \& Zhang 1998). Among them, parameter $\left\langle h_{\|}>=<\mathbf{B}_{\|} \cdot(\nabla \times \mathbf{B})_{\|}\right\rangle$ calculated in the photosphere reflect mean twist of an active region magnetic fields, the negative denoting left-handed twist and the positive denoting righthanded twist. However, what is the overwhelming origin of twist of magnetic fields. And, is the origin related to the tilt of magnetic polarity axis? In another word, whether does the twist of magnetic fields occur due to Coriolis force?

If the tilt angles are set to be positive/negative for the active regions which adhere to Hale-Nicholson Law in the northern/southern hemisphere, Tian et al. (2000a) found that almost $60 \%$ of bipolar active regions have negative correlation between the sign of the tilt angle and the sign of the twist parameter $\left\langle h_{\|}\right\rangle$. They speculate that the twists of magnetic fields are produced in convection zone. However, there are still other three possibilities: Coriolis force, convection turbulent motions and different rotation. Tian et al. (2000b) compared the quantity of the twist produced due to Coriolis force and turbulent motions on the basis of model equations (Log cope \& Klapper 1997; Longcope et al. 1998) and found the effect of Coriolis force is much larger than turbulent motions.

In present paper, we find some observed evidences on the twist of magnetic fields resulted from Coriolis force. First, the averaged tilt depends on the latitude, that is agreed with the previous results. The averaged twist depend on the latitude similarly to the tilt. Second, the quantity of the tilt are decreased with 
the separation of magnetic polarity (Tian et al. 1999). So are the twist. The
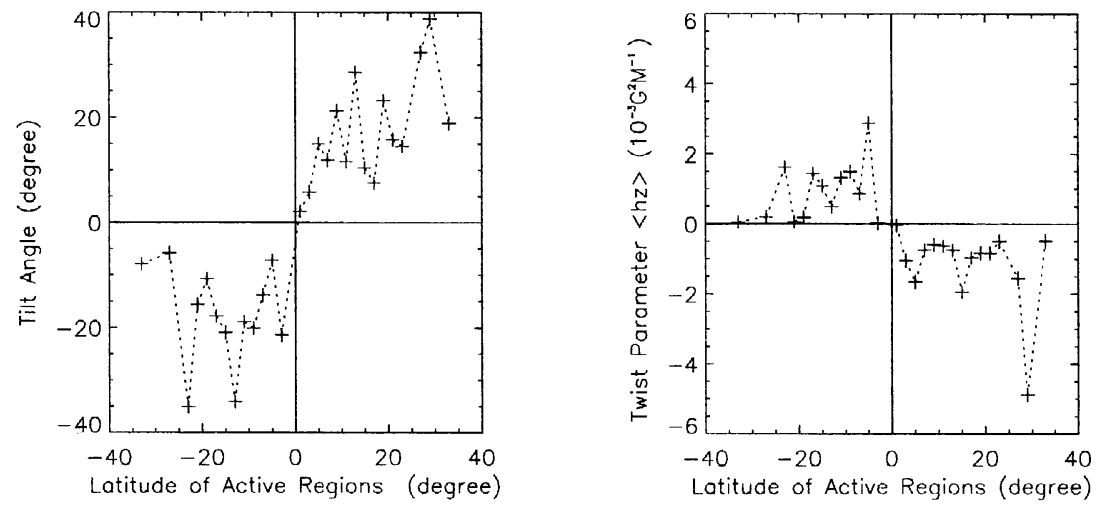

Figure 1. The tilt angle and the twist parameter with the latitude. Both are averaged in 2.0 degree interval of the latitude.
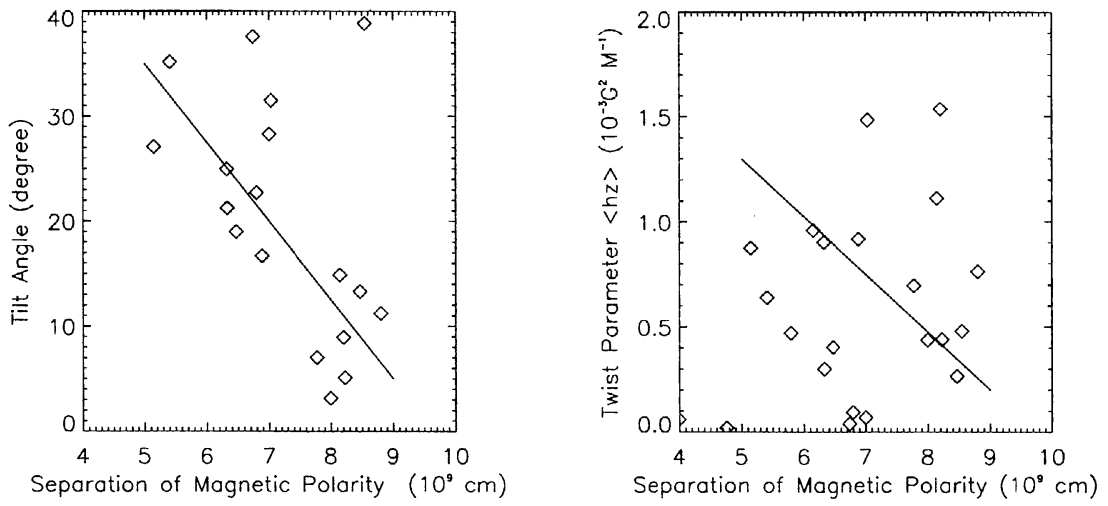

Figure 2. The tilt angle and the twist parameter with the separation. They are averaged in 2.0 degree interval of the tilt.

quantity of the tilt is decreased with magnetic flux. But, the twist is increased with magnetic flux. Why is it? we need to study it in the future. Third, the quantity of the mean twist varies with time similarly to the mean tilt angle.

From results above, we know the variation of the twist almost follows that of the tilt angles. Therefore, we deduce that the origin of the twist is the same as the tilt. In another word, Coriolis force is also the overwhelming origin of the twist of magnetic fields occurred in solar atmosphere.

\section{References}

Babcock H. W. 1961, ApJ 133, 572

Bao S. D. \& Zhang H. Q. 1998, ApJ 496, L43

Bao S. D. Pevtsov A. A., Wang T. J., Zhang H. Q. 2000, Solar Phys. in press Fisher G. H., Fan Y. \& Howard R. F. 1995, ApJ 438, 463 

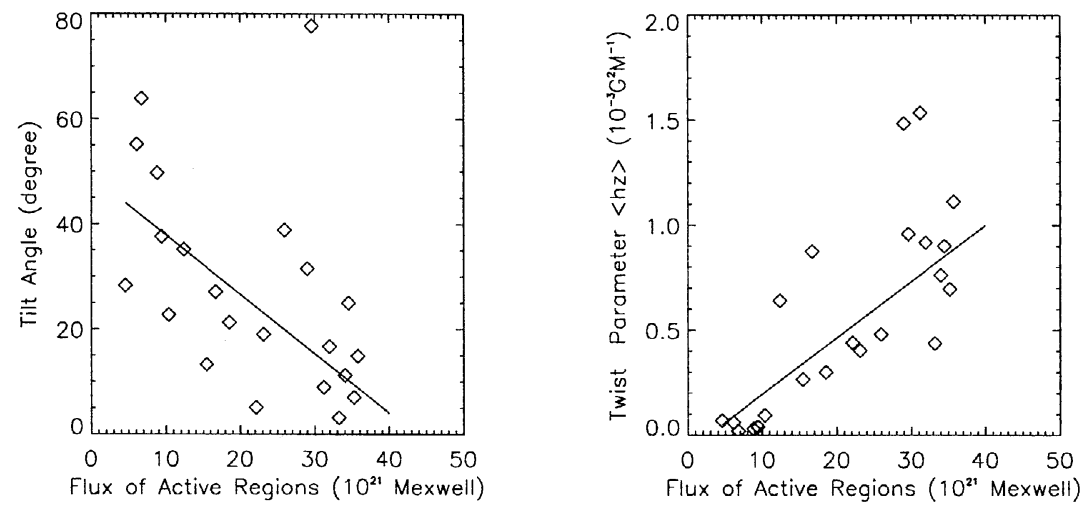

Figure 3. The tilt angle and the twist parameter with the magnetic flux. They are averaged in 2.0 degree interval of the tilt.
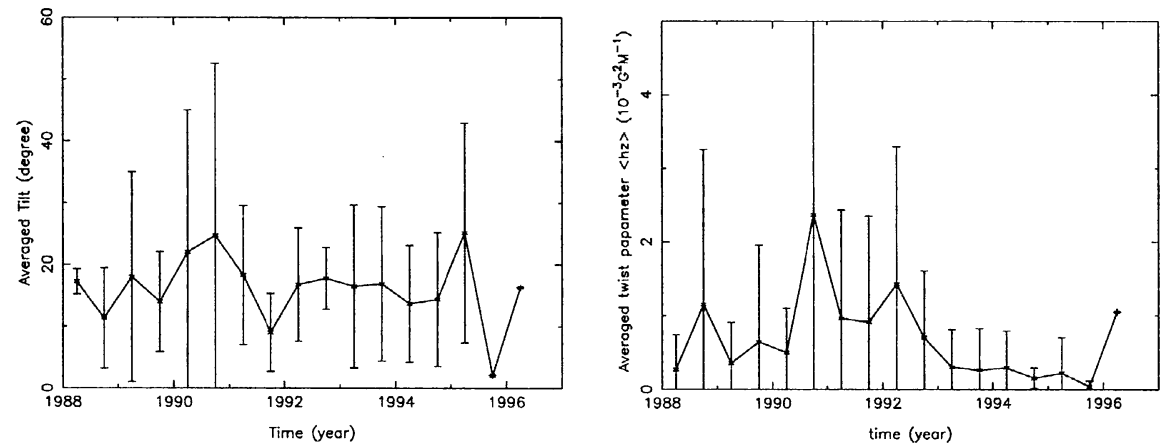

Figure 4. The tilt angle and the twist parameter with time. Both are averaged in 0.5 year interval of time.

Hale G. E., Ellerman F., Nicholson S. B. \& Joy A. H. 1919, ApJ 49, 153

Howard R. F. 1991, Solar Phys. 132, 49

Longcope D. W., Fisher G. H. \& Arendt S. 1996, ApJ 464, 999

Longcope D. W. \& Klapper I. 1997, ApJ 488, 443

Longcope D. W., Fisher G. H. \& Pevtsov A. A. 1998, ApJ 507, 417

Martin S. F., Billamoria R. \& Tracadas P. W. 1994, In Solar Surface Magnetism, eds. R. J. Rutten \& C. J. Schrijver(Dordrecht: Kluwer), 303

Pevtsov A. A., Canfield R. C. \& Metcalf T. R. 1995, ApJ 440, L109

Rust D. M. \& Kumar A. 1996, ApJ 464, L199

Tian L., Zhang H., Tong Y. \& Jing H. 1999, Solar Phys. 189, 305

Tian L., Bao S., \& Zhang H. 2000a, A\&A submitted

Tian L., Bao S., \& Zhang H. 2000b, Solar Phys. submitted

Wang J. X., Shi Z. X., Wang H. N., Lü Y. P. 1996, ApJ 456, 861

Wang Y. M. \& Sheeley N. R. 1991, ApJ, 375, 761 